\title{
PATENTS
}

\section{Recent patent applications in the area of peptides}

\author{
Patent \# \\ Subject \\ New Leishmania acidic ribosomal P-protein family \\ polypeptide. Used to develop products for diagnosis, \\ detection and protection against Leishmania infections. \\ WO 9633405 \\ Sample preprocessor for analytical and preparative \\ chemical analysis. Facilitates reliable and reproducible \\ separation of small amounts of analytes. \\ WO $9633271 \quad$ New mutant allele(s) of the BRCA1 gene. Useful \\ for assessing susceptibility to inherited breast \\ and ovarian cancer. \\ WO $9632955 \quad$ Neuropeptide Y (NPY) analogs. Useful for treating \\ hypertension. Includes amino acids 28-35 of human \\ NPY, but with D-Thr at position 32. \\ US 5567682 Treatment of Alzheimer's disease by intranasal \\ administration of peptide, e.g., peptide T.
}

WO $9632477 \quad$ New genes for murine lymphocyte-specific interferon regulatory factor. Used for modulation of lymphocyte activation and proliferation.

WO 9632475 DNA binding proteins with additional zinc finger domain. Having altered specificities, used to regulate gene transcription for production of specific polypeptide(s).

WO 9632412 Peptide(s) that suppress the phosphorylation of $\lambda-\kappa-$ $\beta-\alpha$. Act as effective antiinflammatory and immunosuppressant agents.

WO 9632411 Synthetic peptide(s) comprising phosphotyrosine binding domains. Used in diagnosis, screening, and therapy of, e.g., breast cancer.

WO $9632410 \quad$ Novel tetra- and pentapeptide compounds containing two tyrosine residues. Useful as plant growth agents, particularly for monocotyledons such as asparagus, rice plants, and corn.

WO $9632409 \quad$ Novel tetra- or pentapeptide containing two sulfated tyrosine residues. Useful as growth promoter for liliaceous plants such as asparagus, lily of the valley, etc.

WO $9632407 \quad$ Purifying insulin-like growth factor I using cation exchange, hydrophobic interaction, and reverse phase chromatography matrices.

WO 9632406 Purifying artificial polymer exhibiting reversible inverse temperature transition, specifically bioelastic polymers from complex mixtures of proteins, e.g., those found after expression in host.

WO 9632140 Artificial veto cells that inhibit or kill specific T cells. For immunotherapy of transplant rejection, inflammation and autoimmune disease, also related cell membranes and lipid-modified polypeptide(s).

WO $9632132 \quad$ New vaccines for filarial parasite infection(s) comprising C-terminal $\beta$-tubulin amino acid sequence from a parasite.
Assignee

$\begin{array}{ll}\text { Assignee Author } & \begin{array}{l}\text { Publi } \\ \text { date }\end{array}\end{array}$

Corixa Corp

Seattle, WA

Mayo Foundation

Rochester, MN

University of

California

University of East

Carolina

Greenville, NC

Advanced Peptides

\& Biotechnology Sci

Stony Brook, NY

Amgen Canada Inc

Mississauga, Ontario

University of

Washington

Seattle, WA

Chugai Seiyaku KK

Tokyo

Kavanaugh WM, Williams LT

Kyowa Hakko Kogyo KK

Tokyo

Kyowa Hakko

Kogyo KK

Tokyo

Cephalon Inc

West Chester, PA

Bioelastics Res Ltd

UAB Res Found

Birmingham, AL
Reed SG

Benson LM, Braddock WD, Naylor S, Oda RP, Tomlinson A

Friedman L, King M, Lynch E, Ostermeyer B, Rowel S, Szabo C, Lee M

Leonard SA, Nyce JW 10/24/96

Pert CB

$10 / 22 / 96$

A

Grossman A,

$10 / 17 / 96$

A1

Richardson $C D$

Cheng C, Young ET

$10 / 17 / 96$

Ishikawa Y, Kuno K,

$10 / 17 / 96$ Matsushima K

Kavanaugh WM,

$10 / 17 / 96$

Williams LT

Matsubayashi $Y$,

$10 / 17 / 96$ Sakagami $Y$

Matsubayashi Y,

$10 / 17 / 96$

Sakagami $Y$

Abrams JN, Brierley RA, 10/17/96

Hanson JM, Maslanka FC

McPherson DT, Urry DW, 10/17/96

Xu J

Kaplan DR Tykocinski ML

$10 / 17 / 96$

McGill University Montreal, Quebec Upjohn Co

Bughio N, Faubert GM, 10/17/96

Kalamazoo, MI

A1

2

1

A

A1 